\title{
Palmengarten 2007
}

\author{
Matthias Jenny
}

Januar: Exotische Schmetterlinge sorgen auch zum Jahresbeginn 2007 wieder für ein verlockendes Kontrastprogramm im Palmengarten. Mitten im Winter flattern die geflügelten Schönheiten in der zum zweiten Mal veranstalteten Flugshow durch die Galerie am Palmenhaus. Wer dem Winter entfliehen und sich vom Festtagsstress erholen will, kann hier in den tropisch warmen Schmetterlingshäusern auf Entdeckungstour gehen. Auch die Grüne Schule widmet ihre Veranstaltungen für Kinder den faszinierenden Lebewesen. Seit dem 20. Oktober lockte die Schau schon 46000 Besucher an. Erfreulich auch die Gesamtstatistik des eben vergangenen Jahres: 2006 zählte der Palmengarten 805000 Besucher - ein Rekordergebnis.

Mit bis zu 12,4 Grad sorgt der Januar für vorzeitige Frühlingsgefühle, denn schon sprießen Schneeglöckchen und Krokusse aus dem noch mit vorjährigem Laub bedeckten Boden. Die traditionelle Kamelien-Ausstellung, die am 12. Januar eröffnet wird, ist nicht nur für KamelienFreunde ein Muss. Die Schau markiert den ersten Höhepunkt der Blütenschauen im Palmengarten.

Hinter den Kulissen werden indes nicht nur eifrig Besucher und Pflanzen gezählt: Die Stadt Frankfurt stellt ihr veraltetes Buchhaltungssystem auf doppelte Buchführung um - und alle Ämter, der Palmengarten inklusive, müssen ihr Vermögen neu berechnen und bewerten.

Aus Heidelberg erhält der Palmengarten einen vierjährigen Trauerschwan (Cygnus atratus) geschenkt - als Ersatz für den vom Fuchs geholten Partner seines Schwanenpaars, das seit vielen Jahren zu den tierischen Bewohnern des Großen Weihers gehörte. Obwohl das allein gebliebene Weibchen schon 16 Jahre alt ist, sorgt der reichlich junge Gatte in spe für keinen Schwanengesang, sondern für berechtigte Hoffnungen auf Nachwuchs.

Abb. 1: Weiße Baumnymphe, einer der auffälligsten und größten Schmetterlinge in der Flugshow.
Die Zukunft des Gesellschaftshauses ist gesichert: Ende Januar erteilt die Stadt die Baugenehmigung zur Sanierung des historischen Bauwerks. Die veranschlagten Kosten betragen 29,2 Mio. Euro. Das Land Hessen wird davon 75000 Euro übernehmen. Um den prachtvollen Festsaal wiederherzurichten, werden 2,5 Mio. Euro veranschlagt, mit 6,5 Mio. Euro soll die Haustechnik modernisiert werden. Das Architektenbüro David Chipperfield (Berlin, London) stellt der Öffentlichkeit die geplanten Bauabschnitte im Einzelnen vor.

Februar: Während die Besucher sich noch am fernöstlichen Kamelien-Zauber und den tropischen Schmetterlingen erfreuen, laufen im Hintergrund schon die Vorbereitungen zur alljährlichen Garten-Messe auf Hochtouren. Die allseits erhoffte Verlängerung der Flugshow ist

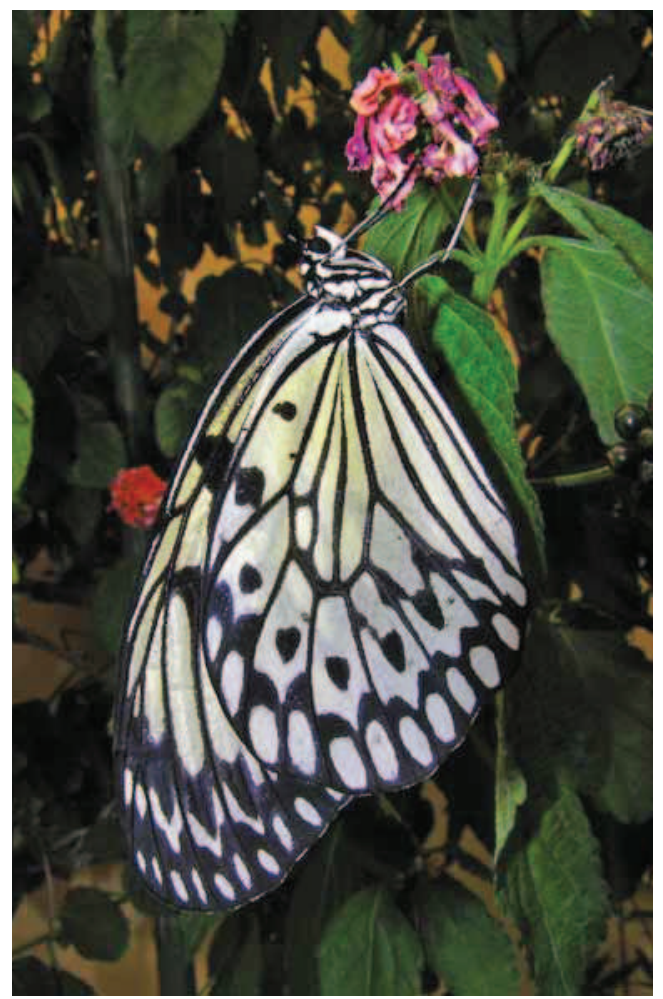




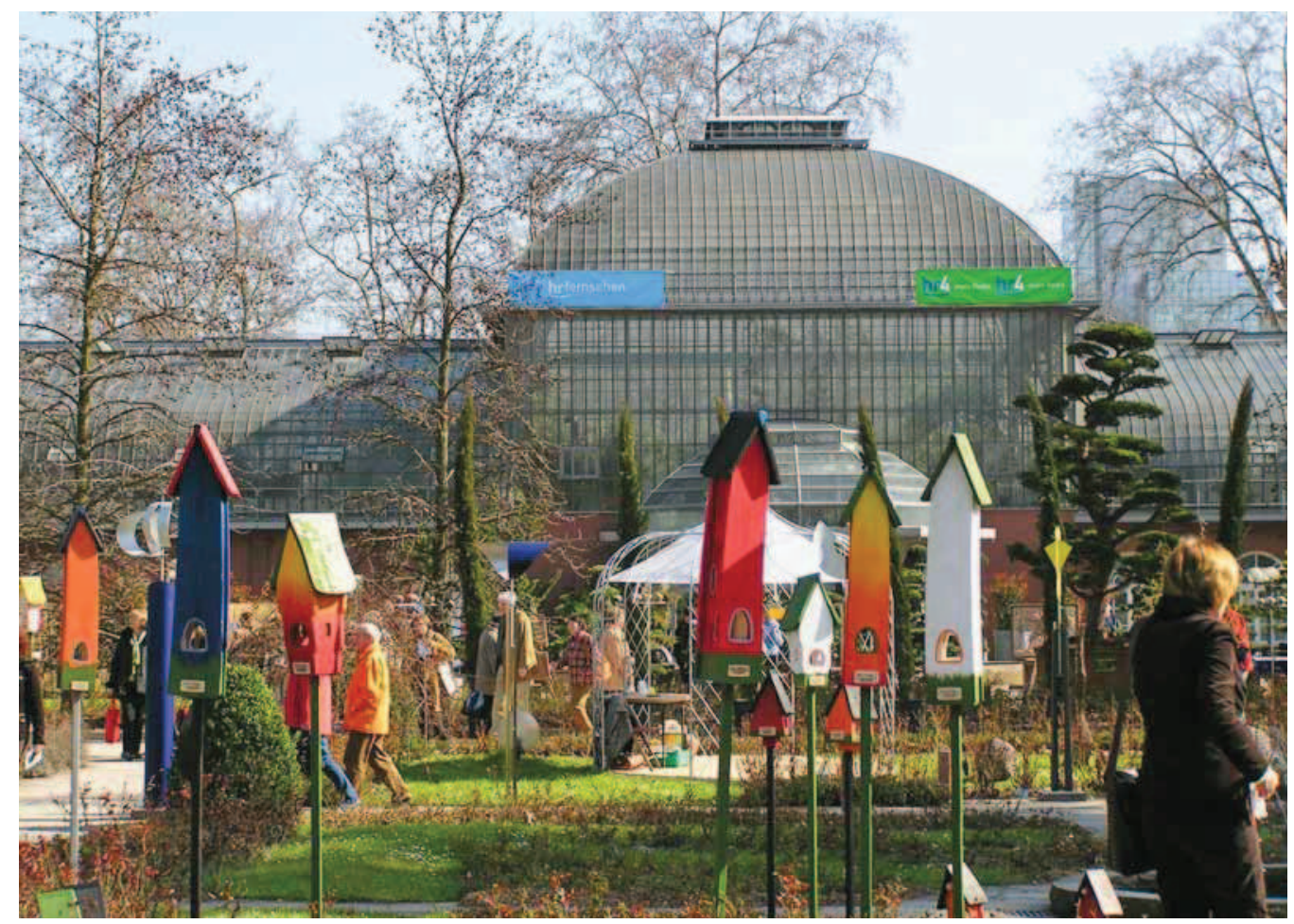

daher nicht möglich. Denn in diesem Jahr präsentiert sich die Informations- und Verkaufsausstellung zum Auftakt der Garten-Saison unter dem neuen Titel „Garten 2007“ mit einem neuen Konzept, bei dem auch die Galerien am Palmenhaus einbezogen sind - eine innovative Schau, die alles Nichtgärtnerische aus dem Angebot verbannt und das Thema Gartenkultur in den Mittelpunkt eines malerischen Marktgeschehens stellt.

85000 Besucher haben in 18 Wochen die insgesamt etwa 4000 Falter der „Flugshow“ bestaunt. Nun finden die verbleibenden Raupen und Puppen der wertvollen Falter im Schmetterlingshaus in Erfurt ein neues Domizil. Kaum ist die Ausstellung am 25. Februar zu Ende, wird in der Galerie am Palmenhaus schon die Frühlingsausstellung eröffnet. Zum betörenden Duft und zur Farbenpracht von Hyazinthen, Tulpen, Narzissen \& Co. unterm Glasdach kommen Außentemperaturen um 15 Grad. So können die Besucher drinnen wie draußen ihre Sehnsucht nach Frühling stillen.
Im „Zeichen der Rose“ veranstaltet der Internationale Women's Club seinen Freundschaftsball. Die Gäste sind eingeladen, mit ihren Spenden den Umbau des Rosengartens im Palmengarten zu unterstützen. 18000 Euro kommen dabei zusammen, die dazu beitragen, das Rosenparterre gegenüber dem Eingangsschauhaus Siesmayerstraße von Grund auf zu erneuern und zu verschönern.

Ein besonderer Festakt schließt den Februar ab: Im Römer wird des 300. Geburtstages von Johann Christian Senckenberg gedacht.

März: Als Abschluss der Frühlingsblumen-Ausstellung gestalten Schüler dreier Grundschulklassen Bilder aus Blüten. Kurz nach Ende der beliebten "Flugshow" stehen natürlich die Schmetterlinge als Motiv im Vordergrund. Die Helmholtzschule unternimmt indes mit ihren Fünftklässlern einen Nachtausflug ins Tropicarium und lernt dort bei Naturerlebnisspielen die Besonderheiten des Regenwalds kennen.

Abb. 2: Buntes Treiben auf der Gartenmesse. 


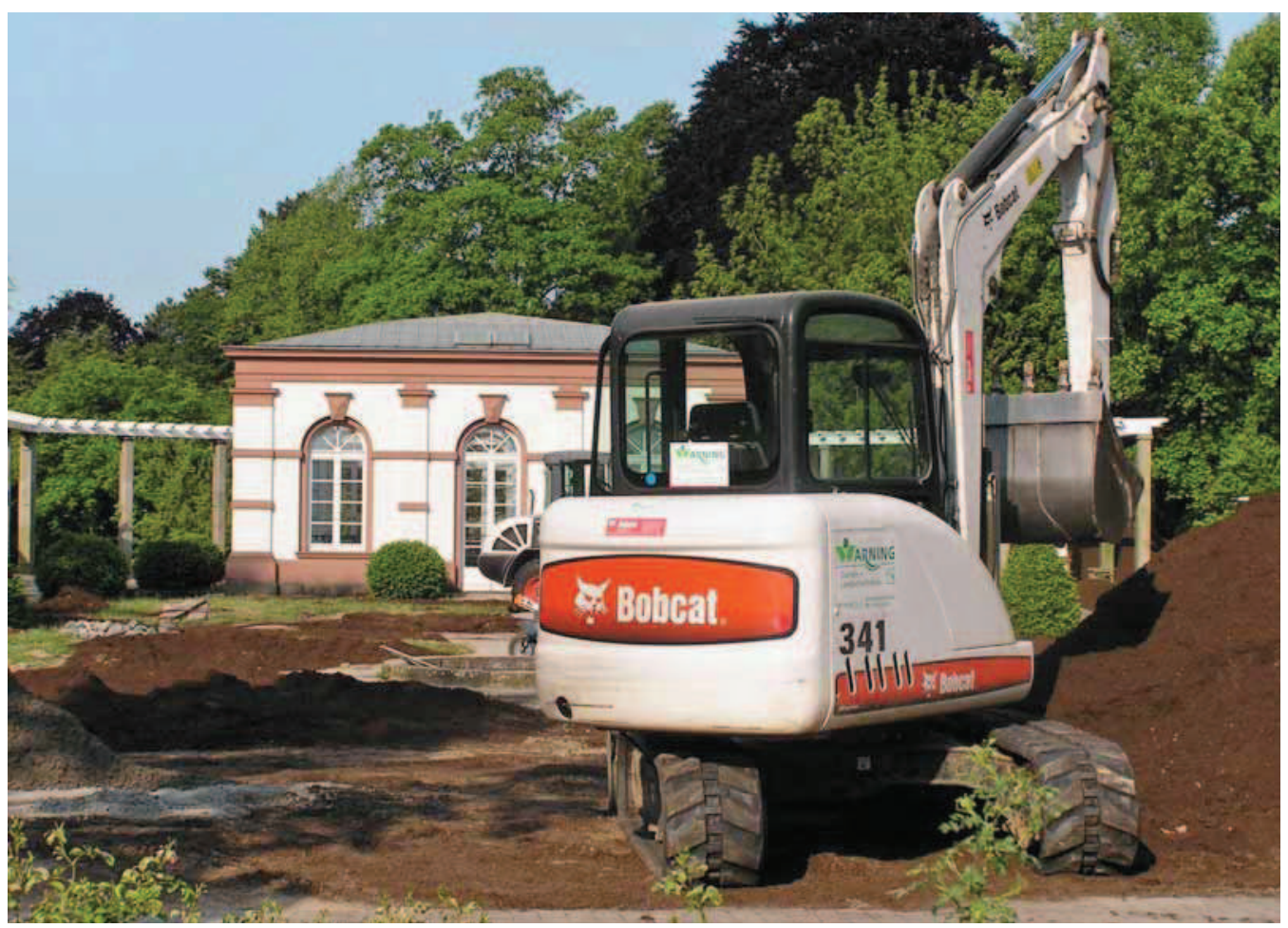

Die nun auch im Freiland zu Abertausenden blühenden Krokusse kündigen unweigerlich den Frühling an - ein rechtes Stimmungsbild also zur Eröffnung der neuen Verkaufs-Ausstellung "Garten 2007“. Mit 90 Ausstellern auf $4000 \mathrm{~m}^{2}$ Fläche, der faszinierenden Pflanzenraritätenbörse und dem lehrreichen „Bio-Frankfurt"-Pavillon zieht die Schau vom 15. bis zum 18. März rund 30000 Gartenfreunde und Hobbygärtner an. Das neue Konzept hat sich, so der Tenor aller, vollauf bewährt und findet auf Anhieb einhelliges Lob und Zustimmung - beim Publikum ebenso wie bei den Ausstellern.

Mit ersten Spatenstichen beginnt im Rosengarten der unabdingbare Umbau. Die umfangreichen Sanierungsarbeiten, bei denen das gesamte Erdreich ausgewechselt werden muss, ziehen sich mehrere Wochen hin.

„Heiraten mit Rosen" lautet das Thema vom 23. bis 25. März während der "Weißen Woche“ im Palmengarten. In Zusammenarbeit mit dem

Abb. 3: Der Rosengarten wird umgestaltet.
Standesamt Frankfurt findet in der Galerie eine Hochzeits-Ausstellung statt. Sie bietet Brautpaaren in spe - und allen, die ein bisschen mitträumen wollen - ein vielfältiges Angebot rund um das feierliche Thema.

Gleich nebenan in der Galerie am Palmenhaus hält die exotische Königin der Blumen Hof, die Orchidee: Wie in jedem Jahr wird die ebenso reichhaltige wie reizvolle OrchideenSchau zum Treffpunkt aller Liebhaber dieser bezaubernden Pflanzenfamilie. Wer ihrem Reiz erliegt, kann die tropischen Schönheiten hier immer auch gleich erwerben.

Hommage an den Palmengarten: Nach ihrem Lieblingsort befragt, verrät CARMEN Renate KöPER bei der Feier ihres 80. Geburtstags, dass dies just der Garten im Westend sei. Kenner wissen warum: Hier hat die Schauspielerin und Autorin die Veranstaltungsreihe „Literatur im Glashaus" mitinitiiert.

April: Wenige Tage vor Ostern erwartet die Besucher des Palmengartens ein Blütenrausch: In der Galerie wird die Azaleen-Ausstellung eröff- 


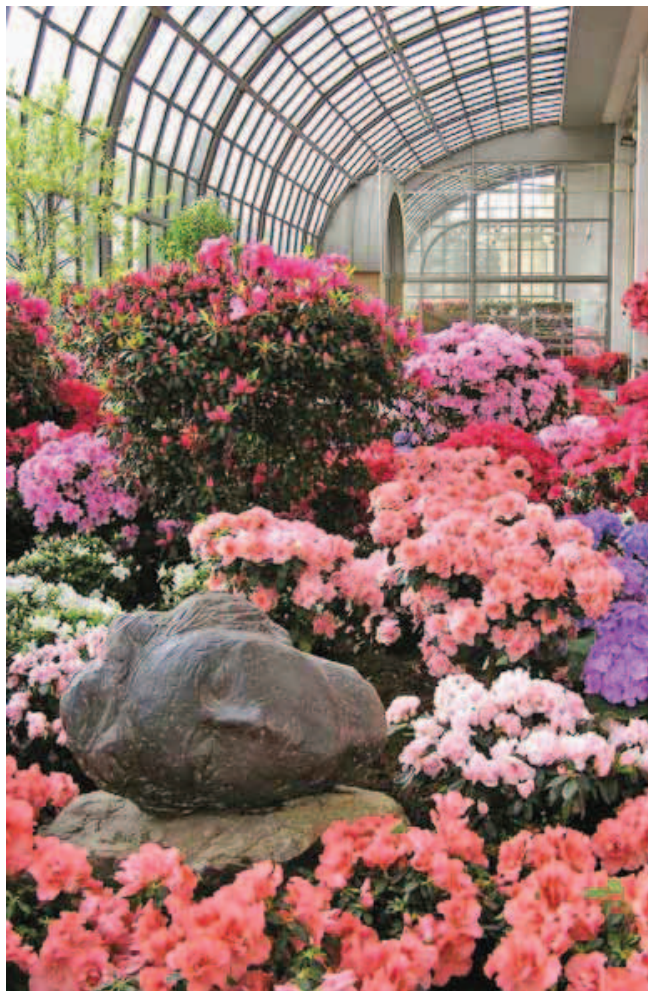

net. Parallel zu den ca. 1000 weiß, rosa, rot und violett blühenden Azaleen der hauseigenen Sammlung zeigt die Künstlerin WANDA PRATSCHKE schwere Bronze-Skulpturen unter dem federleichten Motto „Flora, Eva und die Anderen“.

Hochsommerliche Temperaturen im April locken zahlreiche Besucher in den Palmengarten. Bei Umfragen wird der Park sogar zum schönsten Ort der Stadt gekürt. Bauarbeiten aber sind auch in einem solchen Gartenreich von Zeit zu Zeit nötig. So muss die Palmenhaus-Terrasse für einige Wochen geschlossen werden, um die darunter liegenden Kellergeschosse abzudichten und die Decken der Kellerräume zu erneuern. Auf der Spielwiese wird unterdessen eine Ausstellungshalle von $1000 \mathrm{~m}^{2}$ Größe installiert. Sie ist gleichsam Vorbote der im Mai beginnenden Pyramiden-Ausstellung.

Einen Geburtstag der besonderen Art gilt es zu feiern, denn ohne ihn gäbe es den Palmengarten nicht: Vor 190 Jahren, am 26. April 1817, wurde Heinrich Siesmayer geboren. Der engagierte Landschaftsgärtner und Schöpfer des Frankfurter Hortus palmarum gestaltete seinerzeit auch den Kurpark von Bad Nauheim.

Mai: Im Wonnemonat erleben die Besucher eines der schönsten Schauspiele im Freiland: Die überwältigende Pracht des vielfarbigen Flors im Rhododendrongarten rings um den Großen Weiher zieht Liebhaber dieser ostasiatischen Blütengehölze von nah und fern an.

Bagger im Palmengarten? Aber ja, wenn auch nur zeitweilig. Schweres Gerät ist nötig, um Wege und Beete rund um Haus Rosenbrunn komplett zu erneuern. Wegen der Sperrzone in diesem Teil des Palmengartens finden die standesamtlichen Trauungen nicht im Haus Rosenbrunn, sondern im Tropicarium statt; auch exotische Pflanzen eignen sich als extravagante Kulisse fürs Ja-Wort ...

Grundsteinlegung auf dem Nachbargrundstück: Die KfW errichtet hier ihre sogenannte Westarkade. Der entstehende Bau wird $57 \mathrm{Me}-$ ter hoch werden und einen besonders niedrigen Energieverbrauch garantieren. Die geplante Tiefgarage soll künftig an den Wochenenden auch Palmengarten-Besuchern zur Verfügung stehen. Just da ist das bestehende Parkhaus am Eingang Siesmayerstraße ja bei schönem Wetter oft vormittags schon belegt.

Die Gesellschaft der „Freunde des Palmengartens" hat einen neuen Vorsitzenden: KLAus Urban ist der Nachfolger von Georg ZizKA, der den Vorsitz des gemeinnützigen Fördervereins nach siebenjähriger Tätigkeit aus beruflichen Gründen niederlegte. URBAN, ehemaliger Personaldirektor einer internationalen Bank, ist dem Palmengarten seit langem als ehrenamtlicher Mitarbeiter verbunden. Zusammen mit seiner Frau Helga ist er Übersetzer mehrerer englischer Rosenbücher und Autor verschiedener Gartenbücher.

Pyramiden am Main: Am 10. Mai findet die Eröffnung der diesjährigen großen Sonderausstellung „Pyramiden - Häuser für die Ewigkeit“ statt. In der eigens dafür errichteten Halle auf der Spielwiese werden die Besucher mit 150 eindrucksvollen Exponaten und Installationen

Abb. 4: Blick in die Azaleenschau. 
ins Alte Ägypten und an die Ufer des Nils entführt. Parallel dazu sind im Lesesaal Tapisserien aus Harrania zu sehen. Ein botanischer Pfad mit sechs Stationen zwischen Ausstellungszelt und Tropicarium bietet überdies spannende Informationen zu den Nutzpflanzen der Alten Ägypter, wie Papyrus, Feige und Myrthe, Mastixstrauch, Oliven- und Johannisbrotbaum.

Juni: Wieder verwandelt sich der Palmengarten für Tausende in eine wogende Picknick-Wiese: Seit 1931 wird das Rosen- und Lichterfest gefeiert - und so ist die Rose auch in diesem Jahr vom 7. bis zum 10. Juni die Attraktion. Die traditionsreiche Rosenschau eröffnet Lottofee Franziska Reichenbacher, die zugleich Patin des neu gestalteten Rosengartens ist. Rund 30000 Besucher werden an den vier Tagen der Feier rund um die stachelbewehrte Königin der Blumen gezählt.

„Der Vielfalt auf der Spur" lautet das Motto einer Aktionswoche, bei der das Netzwerk „BioFrankfurt" in Zusammenarbeit mit dem Senckenberg-Museum, dem Zoo Frankfurt und dem Palmengarten den Artenreichtum der Erde mit spannenden Mitmach- und Gewinnspielen vorstellt.

Der Palmengarten-Direktor zu Gast bei Bundespräsident Horst KöHLER: Im Rahmen einer „Woche der Umwelt" im Schloss Bellevue wird er nach Berlin eingeladen. Als Teilnehmer einer Podiumsdiskussion zum Thema „Botanische Gärten - Schaufenster der Forschung" kann Matthias Jenny vor dem Hauptstadt-Publikum quasi live aus dem Frankfurter Palmengarten berichten - und damit auch ein bisschen für den heimischen Pflanzenhort werben ...

„Mehr Licht“: Die berühmten letzten Worte des Frankfurter Dichterfürsten GoEthe bringen die gute Nachricht für die Sanierung des Gesellschaftshauses wunderbar auf den Punkt. Während der Planungsarbeiten für die Baumaßnahmen des historischen Gebäudes wird entschieden, dass auch das frühere Oberlicht im Festsaal in die Restaurierung miteinbezogen wird. Ge-

Abb. 5: Zelt auf der Spielwiese für die Pyramidenausstellung.

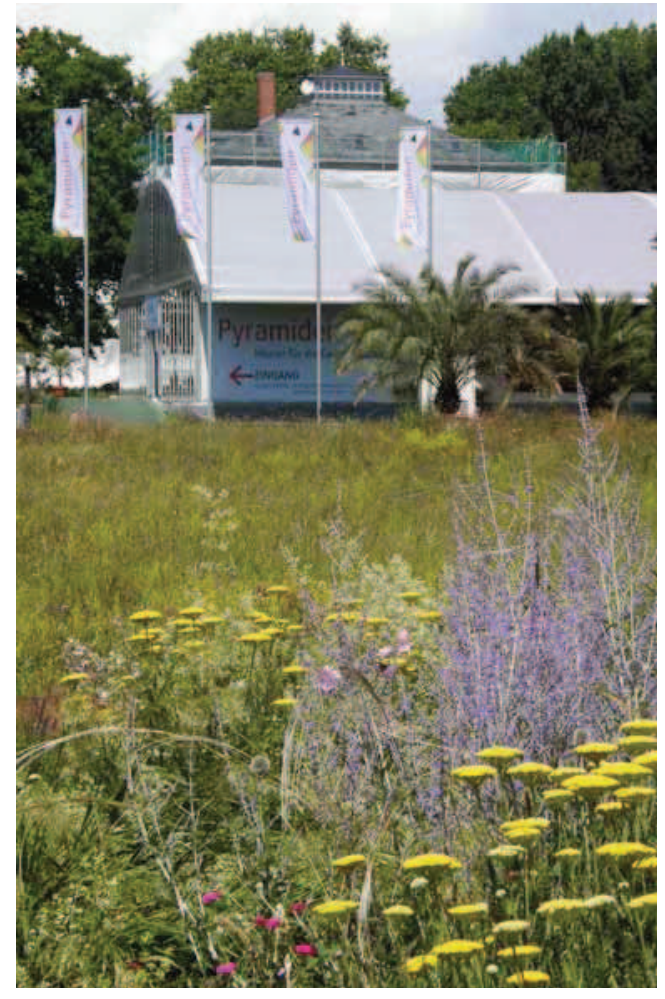

nau das hatten sich nicht nur die Verantwortlichen im Palmengarten, sondern auch viele andere Liebhaber des einst (und bald wieder) schmucken Gesellschaftshauses gewünscht.

Juli: Seit seinen Anfängen ist der Palmengarten ein Treffpunkt für Musikliebhaber. Der Juli ist wieder die hohe Zeit der Open-Air-Konzerte. In der Reihe „Blues im Palmengarten“, die in Zusammenarbeit mit den Friends of Blues, dem amerikanischen Konsulat und der Frankfurter Rundschau veranstaltet wird, sind diesmal Musiker wie Memos GonZales und die Münsterer Bluescasters zu Gast. Die WeltmusikReihe "Summer in the City" präsentiert ebenfalls wieder ein vielfältiges Konzertprogramm. Die Kammeroper feiert ihr 25-jähriges Bestehen mit einer Jubiläumsaufführung der Oper „Ritter Blaubart"von JaCQues Offenbach.

Im Juli wird im Palmengarten freilich nicht nur gefeiert. Wie jedes Jahr legen die jungen Floristen der Philipp-Holzmann-Schule hier ihre praktische Abschluss-Prüfung ab und zei- 


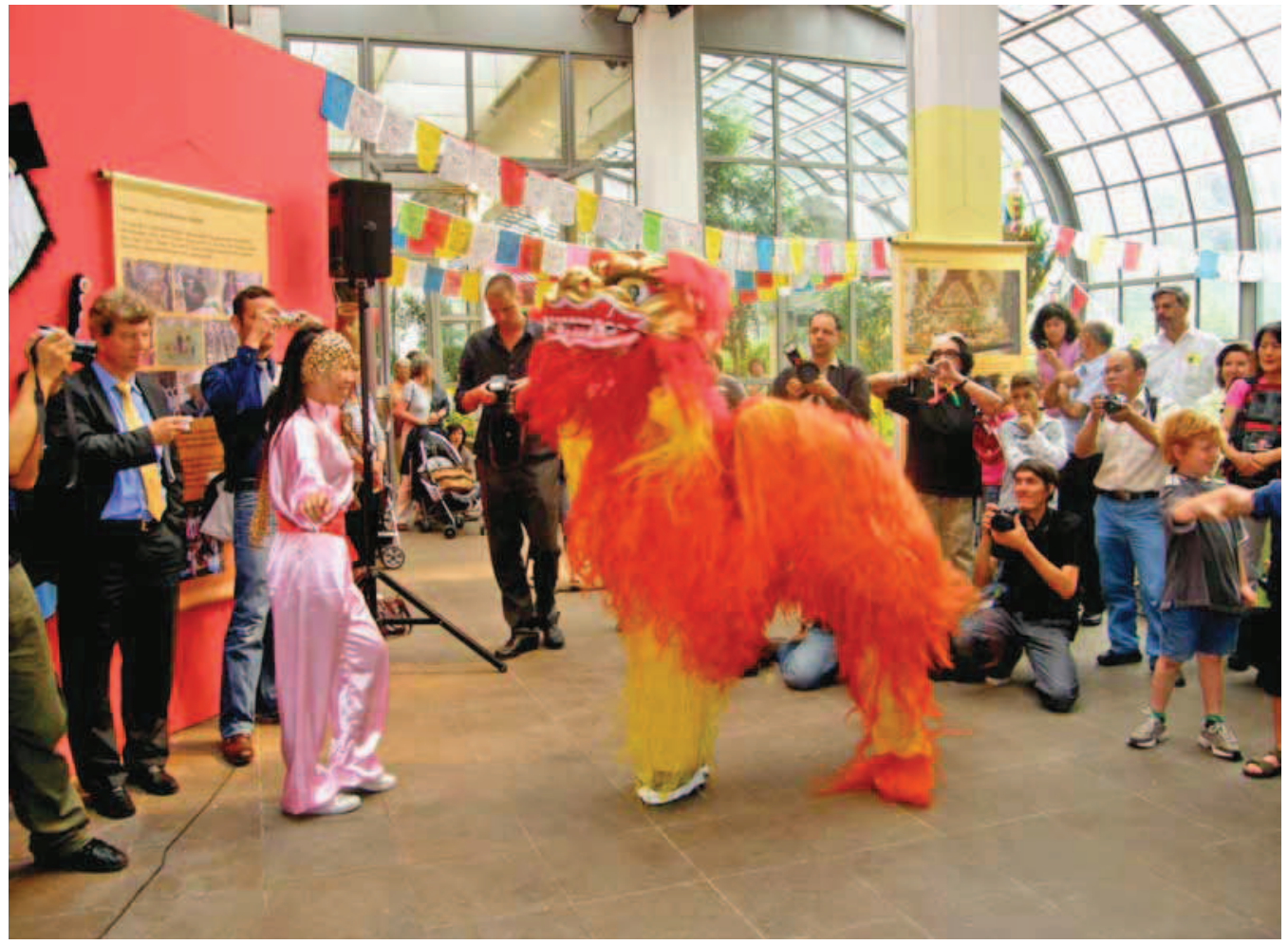

gen ihre floralen Schöpfungen in der Galerie am Palmenhaus.

Schwerpunktthemen der Ausstellung „Pyramiden - Häuser für die Ewigkeit" sind in diesem Monat die Pflanzen Aloe, Hirse und Papyrus.

Rund 400 Jahre ist sie alt, die berühmte Eibe, die 1907, also vor genau 100 Jahren, in einer Aufsehen erregenden Aktion vom einstigen Botanischen Garten in der Senckenberganlage an ihren jetzigen Standort im Palmengarten verfrachtet wurde. Zur Feier des Baums und des eigenen 300. Geburtstags lässt die Senckenbergische Stiftung den Ehrenplatz dieser bejahrten Taxus baccata neu gestalten. Auf einer Informationstafel wird den Besuchern jetzt die Geschichte des Baums, den schon GoEthe gesehen hat, erzählt. Der spektakuläre Transport wurde auch beim Jubiläums-Festakt der Senckenberger in Erinnerung gerufen.

Mit einem „Löwentanz“ wird am 19. Juli die zweite große Sonderausstellung des Palmengartens eröffnet: „Pflanzen und Menschen in Süd-
west-China“ ist sie überschrieben. Die Geografin Sylvia Reinhardt hat in Zusammenarbeit mit dem Palmengartenteam tatkräftig dazu beigetragen, dass in der Galerie am Palmenhaus eine ebenso reich bestückte wie faszinierende Ausstellung über die Vielfalt von Flora und Kultur von Yunnan und benachbarten Provinzen im Südwesten Chinas zustande kam. Als Bestandteil der Ausstellung illustriert eine gesonderte GTZ-Ausstellung in der Galerie die Vielfalt in der Landwirtschaft von Hainan und Hunan. Auch außerhalb der Ausstellungs-Galerie geht es um Südwest-China. Vor dem Galerie-Eingang sind zwei asiatisch gestaltete Gärtchen zu bewundern. Passend zur Ausstellung führt ein Rundgang zu chinesischen Pflanzenschätzen durch den Palmengarten. Eine Fotoausstellung im Tropicarium stellt das „Pflanzenparadies Huanglong" vor.

Abb. 6: Eröffnung der China-Ausstellung mit Löwentanz. 
Die Fraport AG, die als Sponsor die Pyramiden-Ausstellung unterstützt, feiert ein sommerliches Familienfest im Palmengarten.

August: In der Ferienzeit macht der Palmengarten seinem Motto „Ein Tor zur Welt der Pflanzen" wieder einmal alle Ehre: Wo anders kann man im Rhein-Main-Gebiet an nur einem Tag von der Pflanzenwelt Südwest-Chinas bis zu den Pyramiden Ägyptens reisen?

Im Sommer-Sukkulentengarten, einer weit und breit einmaligen Attraktion, wartet man jetzt nur auf richtig heiße Tage, damit sich die Blüten der hier gezeigten Kakteen, Agaven und anderen wasserspeichernden Pflanzen voll entfalten können.

Veritable Pyramidenbauer zu Gast: Im Rahmen eines Projektes „Pro Job“ bauen sieben junge Männer einer Jugendwerkstatt in der Lüneburger Heide die „Knickpyramide“ nach. Zur Anerkennung bekommen sie eine Führung durch die Frankfurter Pyramidenausstellung und sind begeisterte Fahrgäste im Palmen-Express. Im Rahmenprogramm zur China-Ausstellung gibt es unterschiedliche Spezialführungen, die beispielsweise über chinesisches Wildgemüse informieren. Sogar im benachbarten Botanischen Garten der Universität gibt es eine besondere China-Führung.

Mit einer stimmungsvollen "Gala-Nacht" der Opernstars feiert die Kammeroper im Musikpavillon des Palmengartens ihr 25-jähriges Bestehen. Auch das Papageno-Musiktheater kann ein Jubiläum begehen: Seit zehn Jahren hat diese einmalige Bühne für Kinder und Erwachsene ihr Domizil im Palmengarten. Mit der Neuinszenierung von „Manche mögen's heiß" nach dem Film ,Some Like It Hot" von BILLY WILDER wird die Jubiläumsspielzeit im schmucken Theaterzelt am Großen Weiher eröffnet.

Eine Ausstellung zeigt die Ergebnisse eines Meisterlehrgangs am Grünberger Bildungs-

Abb. 7 (oben): Koreanische Floristik.

Abb. 8 (unten): Üppiger Sommerflor auf dem Gelände des Rosengartens.
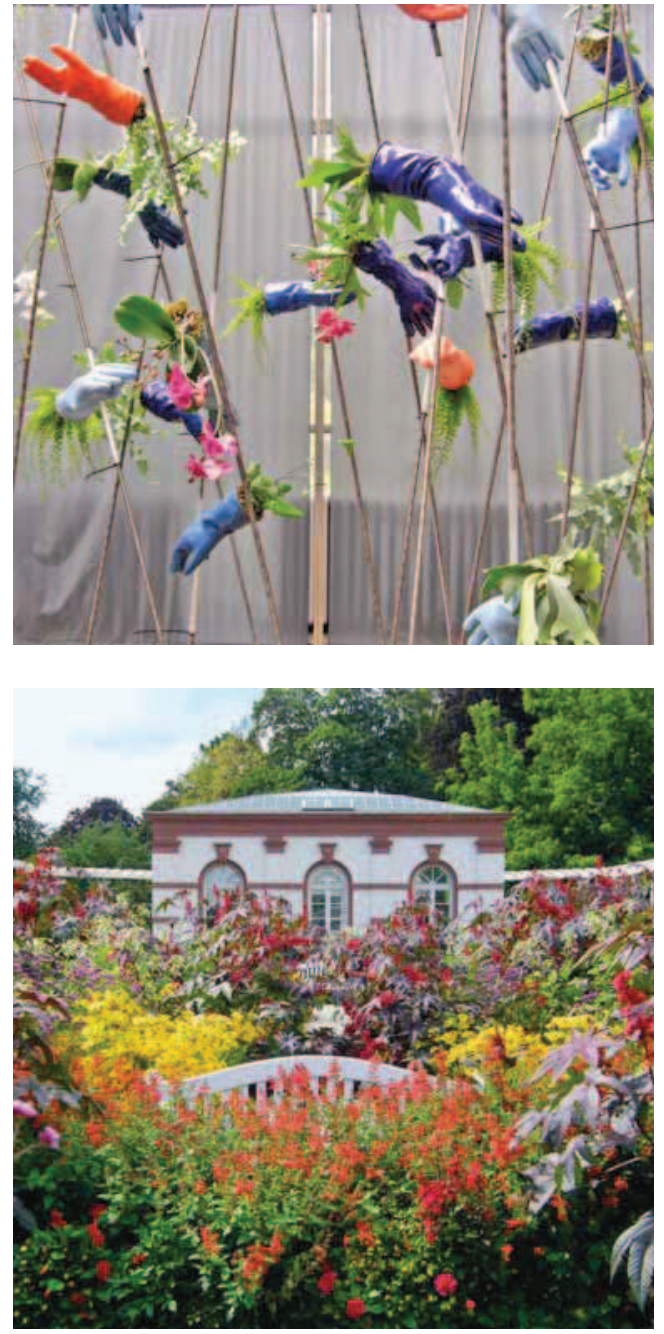

zentrum Floristik. Vierzehn koreanische Floristikmeister zeigen rund 70 Werke, darunter Tisch- und Blumengestecke, Brautschmuck sowie „floralen Körperschmuck“. Die älteste Teilnehmerin des Lehrgangs ist stolze 70 Jahre alt.

An ungewohnter Stelle präsentiert sich der Sommerflor, bei dem sich die Palmengärtner jedes Jahr von neuen Ideen inspirieren lassen. Diesmal erstreckt sich das sommerliche Arrangement mit der Blütenpracht von Tabak und Rizinus in den Beeten am Haus Rosenbrunn.

Seit einigen Jahren schon gibt es eine fruchtbare Zusammenarbeit zwischen dem Palmengarten und der Rhein Main Biokompost GmbH. Um die Besucher auch über die öko- 


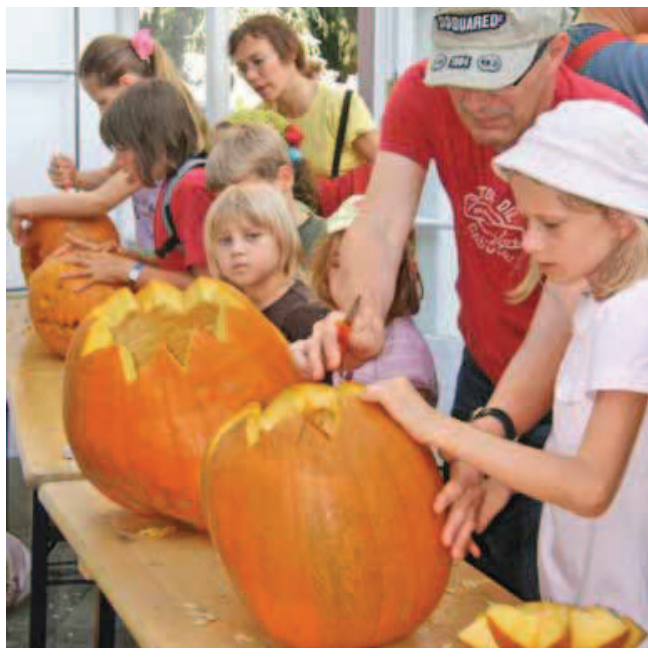

logischen Aktivitäten der städtischen Gesellschaft zu informieren, werden Prospektständer der RMB in den Eingängen Palmengartenstraße und Zeppelinallee installiert. Interessierte finden so auf einen Blick die Broschüren und Veranstaltungsprogramme beider Einrichtungen.

September: Die GWH (Gemeinnützige Wohnungsgesellschaft Hessen $\mathrm{mbH}$ ) unterstützt immer wieder den Palmengarten. Als aktueller Sponsor der Pyramiden-Ausstellung feiert das Unternehmen am 1. September eine „Pyramiden-Party". Zu einer spannenden literarischen Reise durch ein „Rätselhaftes Ägypten“ lädt wenige Tage später die Schauspielerin CARMEN RENATE KöPER ein.

In Bastelaktionen für Kinder geht es im Rahmenprogramm der China-Ausstellung um traditionelle Papierherstellung und chinesische Bilderschrift. Am 6. September lädt die GTZ zu einem Aktionstag „Chinas biologische und kulturelle Vielfalt" mit Vorträgen, Diskussionen und einem abwechlsungsreichen Rahmenprogramm ein.

„BioFrankfurt“, das 2004 gegründete Netzwerk von mittlerweile zwölf namhaften Institutionen, hat sich zum Ziel gesetzt, das öffentliche Bewusstsein für Biodiversität zu steigern. In diesem Herbst startet der Verbund eine breit angelegte Kampagne, bei der mit Führungen,
Ausstellungen, Exkursionen und Vorträgen das Thema vorgestellt und die Artenvielfalt gefördert werden soll.

Der Kürbis, ebenso nahrhaft wie attraktiv und kurios, steht im Zentrum der ErntedankAusstellung. Beim traditionellen Herbstfest im Palmengarten sind Familien, Schüler und alle anderen Besucher zum Schauen, Mitmachen und Genießen eingeladen.

SAKI Kosugi bringt als Gast der Ausstellungseröffnung eine besondere Überraschung mit: Sein Tokyoter Unternehmen für Landschaftsarchitektur möchte in Europa einen japanischen Garten stiften - der Palmengarten sei ihm dazu wärmstens empfohlen worden ...

Krimi-Time im Palmengarten: Für die „Tatort"-Sendung „Unter uns" finden auch in Frankfurts schönstem Garten Dreharbeiten statt. Ausgestrahlt wird die neue Folge der beliebten Fernseh-Krimi-Serie am 14. Oktober.

Königin des Herbstes ist zweifelsohne die Dahlie. Die Vertreter dieser üppigen Blütenschönheit stehen in einem 200 m langen Dahlienband Parade. Der Parcours des attraktiven Herbstflors zieht sich vom Tropicarium bis zur Ausstellungshalle der Pyramiden-Schau.

Anita Albus ist zu Gast im Palmengarten und liest aus „Das Botanische Schauspiel“. Darin wird in 24 Portraits das Schicksal verschiedener Pflanzen erzählt - und das jener Menschen, die sie einst entdeckten: Pflanzenjäger, Forschungsreisende, Botaniker, Gärtner, Maler und Liebhaber.

50000 Besucher haben bisher die Pyramiden-Ausstellung gesehen - keine Frage: die Schau muss verlängert werden und ist daher noch bis zum 21. Oktober geöffnet.

Oktober: Auch im fortschreitenden Herbst sorgt der Palmengarten weiter für Attraktionen. So wird die Orchideenbörse wieder zum begehrten Treffpunkt der passionierten Sammler und Liebhaber dieser edlen Gewächse. Und die Blütenpracht der Chrysanthemen feiert im Ver-

Abb. 9: Kürbisschnitz-Aktion während des Herbstfestes. 
bund mit anderen Blumen des Herbstes in der Galerie am Palmenhaus ihre Hochsaison.

Die Antik Effekten GmbH veranstaltet eine Auktion seltener Aktien im Palmengarten. Bei der Versteigerung der historischen Blätter kommt - als Highlight - auch ein Zertifikat der Palmengartengesellschaft von 1869 unter den Hammer.

November: Ladies first oder: Stadträtin Dr. Manuela Rottmann sowie Lottofee und Rosenpatin Franziska Reichenbacher ziehen sich nonchalant erst einmal Handschuhe über, bevor sie nicht nur symbolisch zum Spaten greifen. Der Anlass: Nachdem im Rosengarten das Erdreich bis auf 70 Zentimeter ausgetauscht wurde, können nun die ersten Pflanzlöcher für die neuen Rosenstöcke gebuddelt werden. Tatkräftig unterstützt werden die beiden Damen bei dieser hoffnungsfrohen Tätigkeit von ANNeMARIE SCHMidT, Präsidentin des International Women's Club, Philipp von Sahr, BMW Niederlassungsleiter, und KLAus Urban, Vorsitzender der Gesellschaft „Freunde des Palmengartens".

Rechtzeitig zum ersten Adventssonntag wird am Freitag, 30. November, die Weihnachtsausstellung eröffnet. Unter dem Titel „Blüten und Gehölze" zeigt sie traditionsreiche Pflanzen zum Fest wie Weihnachtsstern und Christrose, Misteln und Stechpalmen, Amaryllis, Zimt und Nüsse.

Dezember: Die florale Werkstatt der Firma Blumen Paidzior GmbH ergänzt die beliebte Weihnachts-Schau nicht nur mit Exponaten. An den Advents-Wochenenden veranstalten die Mitarbeiter auch floristische Workshops fürs Publikum.

Zum Jahresende schließlich lässt sich auch wieder ein scheuer gefiederter Gast im Palmengarten sehen: der Eisvogel. Der bezaubernde bunte Vogel wird von den Mitarbeitern mit

Abb. 10 (oben): In der Weihnachtsausstellung.

Abb. 11 (unten): In 2007 entwickelte der Affenbrotbaum (Adansonia digitata) im Tropicarium mehrere, recht auffällige Blüten.
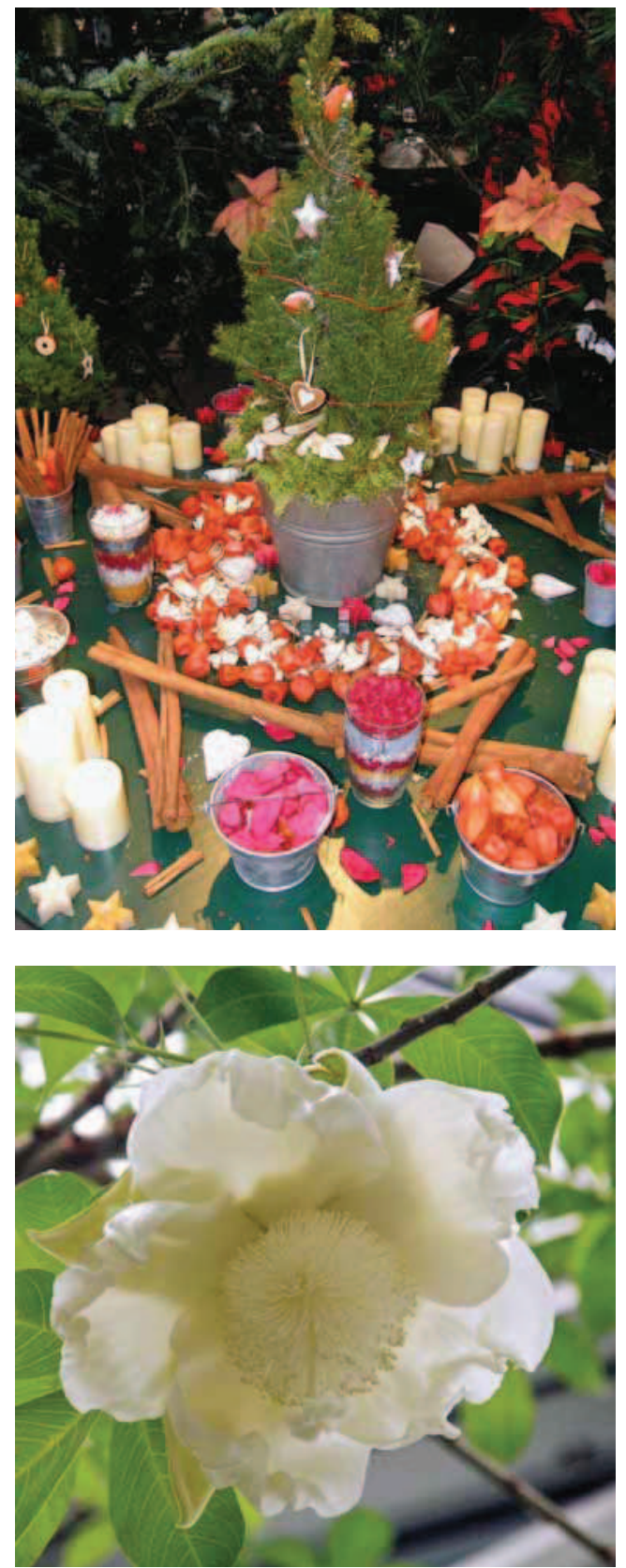

kleinen Fischen versorgt, damit er gut über den Winter kommt - und die Besucher des Gartens während der kalten grauen Jahreszeit mit seiner anmutigen zierlichen Gestalt erfreut. In strengen Wintern ist diese Vogelart mit dem grünblau schillernden Gefieder über der orange leuchtenden Brust besonders gefährdet. 